\title{
Tecnologías de información y comunicación y emprendimiento
}

\author{
Enrique José García Peña
}

Director General. Florida Centre de Formació Coop. V.

egarcia@florida-uni.es

\section{Resumen}

Las tecnologías de la información y comunicación son imprescindibles para la competitividad de los negocios y, por tanto, para iniciar un proyecto emprendedor. La crisis sanitaria ha reforzado la necesidad de digitalización de los negocios y todos estamos cada vez más acostumbrados a interactuar con empresas cuyo nivel de digitalización es cada día más elevado. Este camino hacia la digitalización, en la mayoría de los casos nos ha llegado sobrevenido y uno de cada cuatro negocios han cambiado su modelo de negocio durante la pandemia. Pero ¿estamos realmente preparados para consolidar las tecnologías en nuestros negocios? ¿Responden los perfiles de las personas emprendedoras a las necesidades actuales del entorno? Otro aspecto importante a la hora de emprender es ser consciente del grado de integración de las tecnologías en el proyecto y su relación con la innovación ¿Cómo enfocamos la incorporación de las tecnologías en un proyecto emprendedor? ¿Hacemos que el core business de nuestros proyectos emprendedores se basen en tecnología? ¿O por el contrario incorporamos herramientas tecnológicas a nuestro modelo de negocio tradicional?

\section{Índice}

1. Introducción.

2. El entorno para emprender post pandemia COVID-19.

3. Las TIC y el emprendimiento.

4. Las tecnologías de la información y comunicación y el emprendimiento innovador.

5. El Sistema de Gestión del emprendedor innovador y las TIC.

6. El emprendimiento digital.

7. Conclusiones.

8. Bibliografía. 


\section{Introducción}

El presente artículo no pretende dar la solución maestra para aquellas personas emprendedoras que tengan la necesidad de incorporar tecnología a sus proyectos puesto que, en materia de emprendimiento, todos sabemos que la solución perfecta no existe. Tampoco pretende listar las tecnologías disponibles para las empresas y que facilitan multitud de procesos, funciones, tareas ya que, en cuestión de minutos, dicho listado quedaría obsoleto.

Lo que se pretende es, en base a la experiencia de trabajo con numerosos emprendedores y emprendedoras, poder mostrar la importancia de las tecnologías en los negocios y aspectos a tener en cuenta para incorporarlas, evitando errores que otros han cometido.

Vivimos en un momento social y económico especial puesto que todavía colean los estragos producidos por la crisis sanitaria de la COVID-19. Por este motivo, cobra especial importancia el tema tratado por el papel que han tenido las tecnologías durante los momentos más duros de la pandemia y que han hecho que proyectos empresariales consolidados y proyectos más incipientes, pudieran continuar con su actividad pasando por un proceso de digitalización y de incorporación de las tecnologías de manera forzada, sin orden alguno.

En cualquier caso, lo tratado en el texto a continuación, pretende servir de guía para que las personas emprendedoras puedan establecer estrategias de incorporación de las tecnologías de la información y la comunicación de una manera planificada y que esta estrategia tenga el éxito esperado.

\section{El entorno para emprender post pandemia COVID-19}

Emprender, según la Real Academia de la Lengua Española consiste en "acometer y comenzar una obra, un negocio, un empeño, especialmente si encierran dificultad o peligro". Ya la propia definición contiene términos que advierten de la dificultad de iniciarse en el camino del emprendimiento.

Nunca ha sido fácil emprender, iniciar un proyecto de negocio por cuenta propia, de manera individual o colectiva. Siempre ha sido un arduo camino a nivel burocrático y por supuesto plagado de riesgos e incertidumbres inherentes al propio proyecto. Pero el emprendimiento también ha sido, y es mucho más hoy en día, un medio para salir de las crisis económicas, reducir los niveles de desempleo y dotar de dinamismo a la economía.

Es por ello que el emprendimiento se encuentra en las agendas de las administraciones públicas de cualquier nivel como un elemento a impulsar a través de estrategias claramente definidas, políticas públicas, programas e incentivos a la creación de empresas. Así se recoge en la definición y desarrollo por parte del gobierno de España a principios del año 2021 de la Estrategia España Nación Emprendedora, que sienta las bases del camino que se debe seguir para conseguir el desarrollo de un ecosistema emprendedor innovador. La Estrategia España Nación Emprendedora se define como una estrategia que se "articula para hacer realidad una visión: que para el año 2030 España sea una Nación Emprendedora que haga del 
emprendimiento innovador el rompehielos de un nuevo modelo de país". (Estrategia España Nación Emprendedora, Alto Comisionado para España Nación Emprendedora, 2021).

Dicha estrategia sitúa, como elemento fundamental para su desarrollo y para la consecución de los objetivos planteados, la necesidad de activar un emprendimiento innovador, un emprendimiento que sea extraordinariamente productivo y eficiente y esto solo se consigue a través de negocios en cuyo ADN se encuentren las tecnologías de la información y las comunicaciones.

Esta y cualquier estrategia encaminada al fomento de la actividad emprendedora cobra más importancia en la situación actual del entorno. La pandemia de la COVID19 ha afectado de manera muy importante a la economía y son numerosas las empresas consolidadas y los proyectos emprendedores incipientes que han visto su actividad seriamente condicionada por la crisis sanitaria.

Según el Informe "Situación del emprendimiento en España ante la crisis del COVID19" elaborado por el Observatorio del emprendimiento en España, durante la época más dura de la pandemia, el $42 \%$ de los negocios encuestados cesaron su actividad temporalmente o de manera definitiva. Esto no debería sorprendernos porque la grave crisis sanitaria que hemos sufrido ha impactado en la sociedad a todos los niveles.

Pero si bien esta situación debería ir cambiando a medida que los efectos de la pandemia van disminuyendo y la actividad económica se va regenerando, hay que destacar que el 78,8 \% de los emprendedores miran al futuro con pesimismo y valoran sus expectativas de futuro como negativas.

Este es un dato preocupante y que muestra la necesidad de adaptar los modelos de negocio a la situación del mercado actual y aquí es donde entra la digitalización con un papel fundamental para poder lograrlo.

\section{Las TIC y el emprendimiento}

El uso de las TIC impacta en el desempeño de las empresas de varias maneras y a través de diferentes canales como la automatización de procesos rutinarios; la accesibilidad a la información; la disminución en los costos de transacción y la facilitación de los procesos de aprendizaje (Pierano y Suárez, 2006)

No cabe duda de que las TIC, en la actualidad son un elemento imprescindible para cualquier persona que quiera emprender un negocio. Las tecnologías están directamente relacionadas con el uso eficiente de los recursos y con el crecimiento, eficiencia y productividad empresarial, sobre todo en las que podemos considerar fases iniciales de cualquier proyecto emprendedor. Cualquier emprendedor necesita buscar información e investigar el mercado en el que va a instalar su proyecto, debe conocer las tendencias del mercado donde se encuentran sus potenciales clientes, debe ser capaz de comunicar el proyecto emprendedor que está iniciando y finalmente debe poder medir el éxito de su idea para tomar decisiones a futuro sobre la continuidad de la misma 
Solo con el apoyo de las Tecnologías de la Información y las Comunicaciones en estos procesos, el resultado va a ser satisfactorio y situarán al proyecto en un buen punto de partida para competir en el mercado.

El uso eficiente de las TIC conseguirá obtener ventajas competitivas, pero es necesario tener un plan previo, huir de la incorporación de las tecnologías de manera desordenada sin analizar lo que se necesita y el valor que va a aportar al negocio puesto que las tecnologías no provocan el mismo efecto en todas las empresas, sino que hay condicionantes, como el tamaño de la empresa, el sector de actividad, el mercado objetivo, que marcarán el tipo, el grado y el alcance de las tecnologías a implantar.

Lo que sí es indudable es que son beneficiosas e indispensables a la hora de iniciar un proceso de emprendimiento. Ya se ha mencionado anteriormente el apoyo que supone en etapas iniciales de un proyecto emprendedor pero estos beneficios se extienden durante las fases del desarrollo el negocio.

Las TIC pueden ser utilizadas en cualquier etapa de creación y desarrollo de un negocio y permiten:

- Obtener información del sector y del mercado objetivo: reduce costes en su obtención, tiene mayor alcance y facilita su transmisión. Esto facilita la toma de decisiones al conocer mejor la realidad del entorno, tendencias de mercado y la opinión de los clientes.

- Abarcar más posibilidades de negocio: los productos o servicios de la empresa llegan a un mercado más amplio.

- Mejorar la eficiencia de los procesos, así como la productividad, simplifica los procesos de gestión: el uso de herramientas tecnológicas facilita procesos tales como la planificación, seguimiento y control del negocio, así como otras funciones tales como la gestión contable y la organización del tiempo.

- Mejorar la comunicación con clientes, proveedores, colaboradores o inversores.

- Dar un servicio más inmediato y adaptado a los clientes: es mucho más fácil hacer un seguimiento de los clientes y analizar sus procesos de compra, satisfacción con los productos y/o servicios de la empresa y así poder tomar decisiones en cuanto a estrategias comerciales.

- Mejorar la comunicación y las relaciones personales de los trabajadores (comunicación interna): las TIC facilitan la comunicación eficiente entre miembros de un equipo y permite la conexión en todo momento.

Todos estos beneficios son los que las tecnologías pueden aportar al proyecto emprendedor, pero sin duda alguna hay un elemento fundamental en cualquier proceso emprendedor y se trata de la persona promotora. Esta figura debe tener un perfil, unas características y unas competencias adecuadas para liderar estos procesos de integración o transformación digital en su proyecto emprendedor. 
Una de las primeras tareas del emprendedor es concienciarse de que la tecnología le va a ayudar en su proceso, pero es muy importante no caer en el error de querer incorporar tecnología únicamente sin una planificación y un análisis de necesidades previo. El emprendedor debe ser consciente del cambio que va a suponer la incorporación de tecnología en los procesos de su negocio y debe establecer una estrategia clara, estableciendo necesidades, objetivos y prioridades.

Esta planificación es necesaria porque, la tecnología va más allá de estar presente en las redes o implantar un software determinado. Las TIC pueden y deben introducirse en diversas áreas de la empresa si se quiere realmente aprovechar las ventajas que nos ofrece, y para saber cuáles de ellas lo necesitan es imprescindible hacer esta planificación con un estudio previo de necesidades. Aprendamos de los errores de los demás. El índice de fracaso en la incorporación de la tecnología sin una buena planificación y sin analizar en qué procesos de la empresa existen realmente necesidades, llega a alcanzar el 90\%.

Uno de los motivos por los que implantamos procesos tecnológicos en los negocios es porque las herramientas que hay hoy en día en el mercado nos ayudan a reducir costes, pero, sin este análisis y planificación previa, esta premisa no será real, su efecto será justamente el contrario, un gasto en recursos que en muchos casos los proyectos emprendedores incipientes no se pueden permitir.

Si estableciéramos una hoja de ruta para que el emprendedor pudiera integrar las TIC en su proyecto, un modelo podría ser el siguiente:

1) Analizar e identificar las áreas de mejora de la empresa en materia de integración de las TIC. (DÓNDE)

2) Identificar los beneficios/ahorros que va a suponer en cada una de ellas (POR QUÉ)

3) Explorar las tecnologías disponibles para cubrir cada necesidad. (QUÉ)

4) Analizar la viabilidad de la implantación de las tecnologías, tanto a nivel económico como de aceptación de los usuarios. (CÓMO)

5) Planificar su implantación estableciendo tiempos, prioridades y responsables. (CUÁNDO/QUIÉNES)

6) Control de la implantación.

Solo diseñando un buen proceso, identificando todas las fases desde el análisis a la implantación y control, el emprendedor llegará a alcanzar de manera eficiente, los objetivos que se plantea en su camino hacia la integración de las tecnologías en su proyecto.

Por último, y no menos importante, es que el emprendedor debe ser consciente de que adoptar las tecnologías va a suponer un paso muy importante que va a afectar a la cultura de la empresa, que va a suponer un cambio de paradigma, por tanto, debe contar con el apoyo y la aceptación por parte del equipo con el que cuente. Para ello, es importante una labor de comunicación de todo el proceso hacia las personas que van a ser las usuarias de las tecnologías y establecer un plan de formación en el caso que sea necesario. 


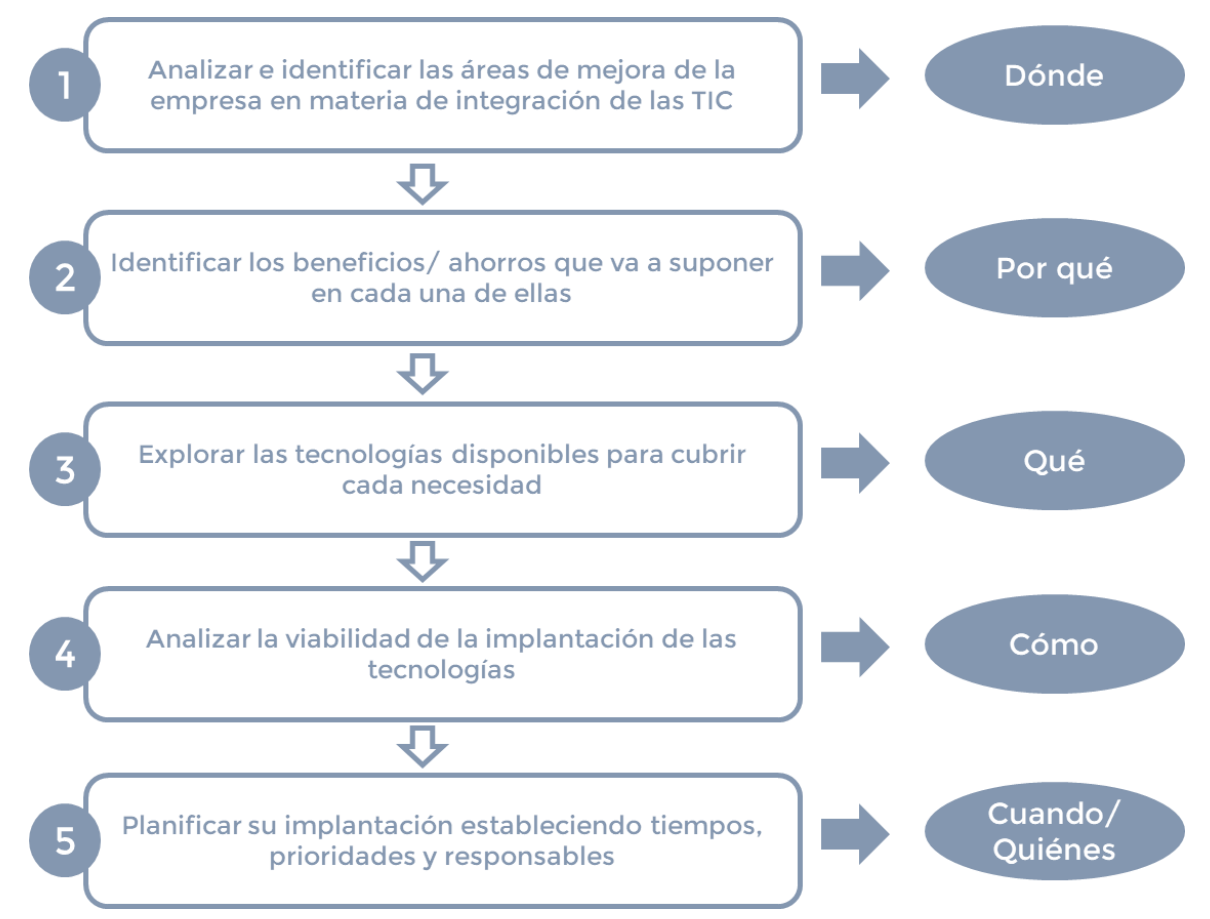

Figura 1: Proceso de planificación e implementación de una estrategia para la incorporación de la tecnología en un proyecto emprendedor/empresarial Fuente: elaboración propia

\section{Las tecnologías de la información y comunicación y el emprendimiento innovador}

Hasta el momento hemos hablado de las TIC y de su importancia en el desarrollo de un proyecto emprendedor, pero ¿sólo con tecnología tenemos asegurado el éxito en los proyectos emprendedores? La respuesta es rotundamente no.

Quizás se pueda pensar que esta cuestión queda alejada del tema principal del artículo, pero no es así. La tecnología se nos presenta como un aliado necesario para llevar a cabo proyectos de emprendimiento, pero la tecnología sin procesos de innovación no sirve para nada. La tecnología sin un buen modelo de negocio, sin un buen análisis de mercado, sin un análisis de viabilidad válido, no va a funcionar. Es por ello que se hace necesario cruzar tecnología, con aquellos procesos de innovación que van a generar un proyecto emprendedor innovador y, por tanto, competitivo.

Debemos entender la tecnología en todos sus niveles como una herramienta de apoyo a los diferentes procesos empresariales. Vistos ya los beneficios que esta aporta, es el momento de pasar a conocer los procesos de innovación que la persona emprendedora debe tener claros para que aquellos productos y servicios que comercialice estén impregnados de un cariz innovador que le permita competir en el mercado con ventaja. De lo contrario, nos podemos encontrar con excelentes 
tecnologías dentro de la empresa, pero con productos y/o servicios con poco valor añadido.

Para ello, es aconsejable que el emprendedor diseñe su propio Sistema de Gestión de la Innovación que, apoyado por las TIC que ha decidido utilizar, le permita generar ideas innovadoras, seleccionarlas, implementarlas y comercializarlas.

Para poder llevar a cabo procesos de emprendimiento innovador apoyado por las TIC, es necesario que el emprendedor posea o desarrolle capacidades de innovación que le permitan:

- captar información que le permita estar al tanto de nuevas oportunidades que el entorno presenta para la empresa.

- dar respuesta ante esta información captada.

- resolver problemas.

- aprender.

- crear e innovar.

- explotar lo aprendido.

Consideramos la capacidad de innovación como el conjunto de características y actitudes que permiten la creación de conocimiento, el aprendizaje y la transferencia de conocimiento, aportando valor al mercado de manera exitosa. Y no solo en cuanto a productos o servicios sino analizar el estado de estas capacidades en otros ámbitos en los que la empresa puede crear valor tales como:

- mercados,

- sectores,

- clientes,

- proveedores,

- colaboradores,

- productos o servicios,

- procesos de la organización y

- los propios trabajadores.

Todas estas capacidades se corresponden con aquellos procesos en los que la tecnología aporta beneficios para el negocio. Desde herramientas tecnológicas que permitan iniciar el análisis de la información del mercado y competidores potenciales, hasta la mejora de procesos de comunicación con proveedores, colaboradores y con los propios trabajadores de la empresa.

Si la persona que va a emprender consigue desarrollar estas capacidades que configuran sus competencias individuales en aquellos ámbitos del negocio en los que puede crear valor (estructura externa e interna del proyecto), únicamente necesitará el apoyo de las TIC adecuadas para desplegarlas de manera eficiente. 


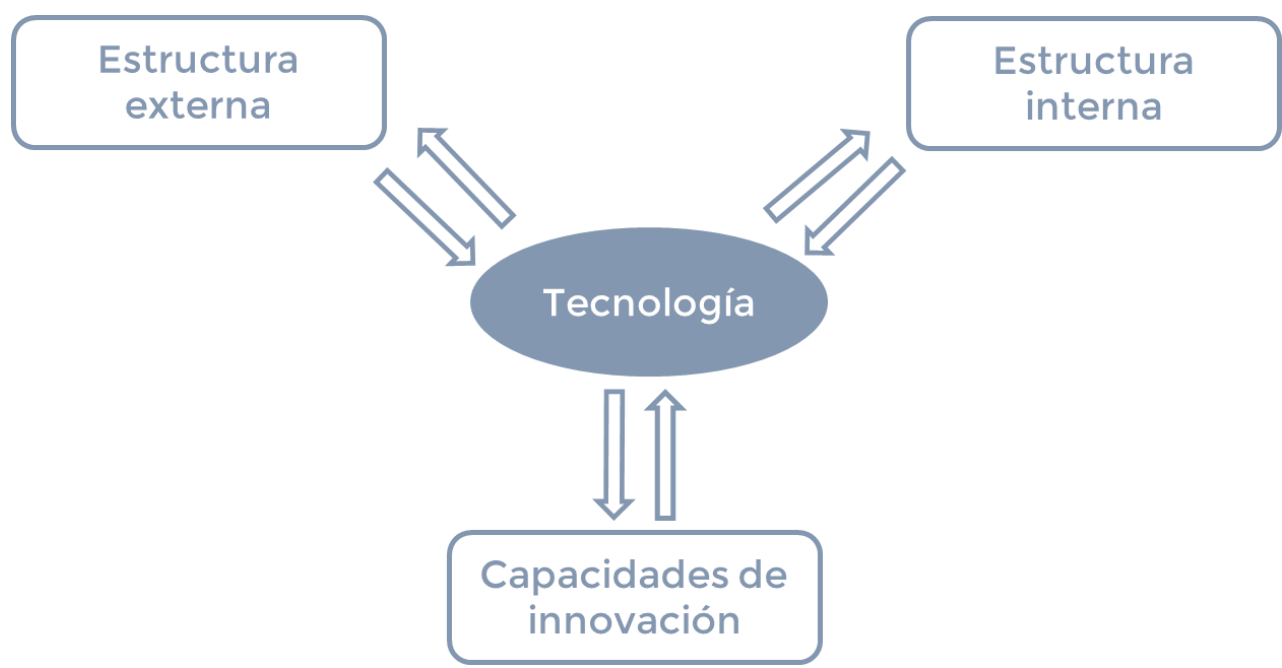

Figura 2: Elementos clave para un emprendimiento innovador Fuente: elaboración propia

Consideramos la estructura externa de una empresa / proyecto, como la relación con los mercados y sectores en los que opera, sus competidores, clientes, proveedores y colaboradores.

La estructura interna incluye los procesos propios de la organización para la generación de productos / servicios.

Las capacidades de innovación incluyen el capital humano de la organización: las competencias, las habilidades, la capacidad intelectual y el conocimiento tácito de los empleados de la compañía.

La tecnología hace referencia al conjunto de recursos necesarios para tratar información a través de dispositivos electrónicos, aplicaciones informáticas y redes necesarias para convertirla, almacenarla, administrarla y transmitirla. A nivel de usuario, sea individual o empresa, las TIC forman el conjunto de herramientas que permiten un mejor acceso y clasificación de la información como medio tecnológico para el desarrollo de su actividad.

La combinación de estos tres elementos clave (estructura externa, estructura interna y capacidades de innovación), junto con el soporte central de las TIC, van a conseguir alcanzar realmente un proyecto emprendedor innovador tecnológico. 


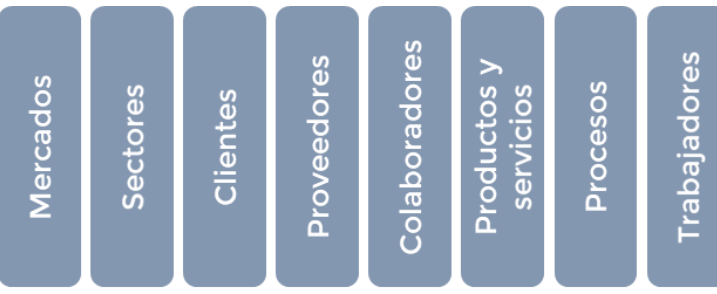

Capacidad de captar información

Capacidad de dar respuesta

Capacidad de resolver problemas

Capacidad de aprender

Capacidad de crear e innovar

Capacidad de explotar lo aprendido

\section{Tecnología}

Figura 3: Matriz de inteligencia organizacional.

Fuente: elaboración propia

Es a partir de este punto desde el que se debe comenzar a construir el propio proceso de innovación, es decir, el proceso que permita pasar de las ideas o señales a verdaderos proyectos de emprendimiento innovadores que puedan ser llevados al mercado con éxito. Las TIC también tienen un papel fundamental en este proceso de gestión de la innovación puesto que también pueden proporcionar apoyo en las diferentes fases. 


\section{El Sistema de Gestión del emprendedor innovador y las TIC}

Un Sistema de Gestión de la innovación es un conjunto de elementos interrelacionados y que interactúan con el propósito de crear valor. Facilita un marco de referencia para que toda la organización desarrolle y despliegue capacidades de innovación, se pueda medir el desempeño y conseguir los objetivos deseados (ISO 56002)

Un Sistema de Gestión de la Innovación con sus procesos de trabajo interno, funciones y responsabilidades de los participantes, etc. debe ser propio de cada organización, es decir, cada empresa debe adaptar el suyo a sus posibilidades, necesidades de innovación, a los ámbitos en los que la empresa desea explorar, al grado de implicación de las personas, etc. pero éste debe ser bien estructurado y organizado, definiendo claramente las etapas o fases por las que van a ser filtradas y trabajadas las ideas, así como las funciones y responsabilidades de cada uno de los miembros participantes.

En definitiva, el emprendedor debe ser capaz de integrar en su proyecto, un sistema que le permita explorar las tendencias en aquellos ámbitos que considere estratégicos, captar nuevas ideas, analizarlas, gestionarlas y darle forma de proyecto de innovación con su posterior implementación en el mercado o a nivel interno en la propia organización. Esto permite establecer un proceso que, apoyado por las herramientas tecnológicas a su alcance permita una constante innovación en los productos o servicios que ofrezca al mercado.

El siguiente modelo de gestión de la innovación muestra de manera genérica las etapas de un proceso de emprendimiento innovador y ejemplos de herramientas tecnológicas que apoyan en las diferentes etapas para convertirlo así en un proyecto realmente innovador. Estos modelos deben ser adaptados a cada uno de los proyectos en función del tamaño y de su tipología. 


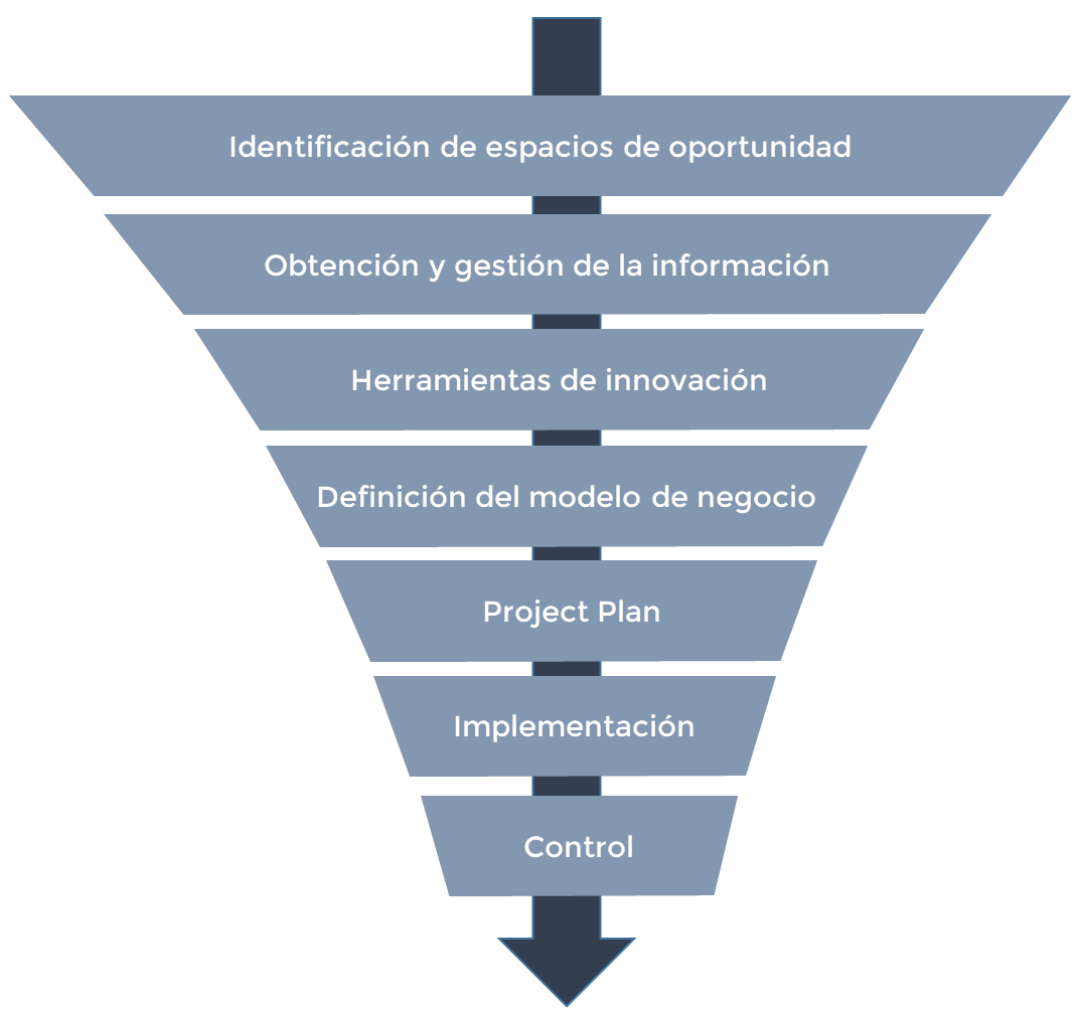

Figura 4: El Sistema de Gestión de la innovación

Fuente: elaboración propia

El propio sistema permite que el emprendedor establezca sus políticas de innovación, así como los objetivos que se marca en este campo estableciendo indicadores que permita evaluar tanto el funcionamiento del sistema como cada uno de los proyectos a nivel individual.

Si cruzamos estas fases del proceso de innovación con las tecnologías que facilitan el mismo y hacen del negocio un proyecto realmente innovador y tecnológico, nos encontramos con numerosas herramientas tales como:

- Herramientas de búsqueda y gestión de la información.

- Herramientas de gestión web.

- Herramientas de trabajo colaborativo.

- Herramientas de comunicación interna y externa.

- Herramientas de marketing y publicidad.

- Herramientas de analítica y posicionamiento.

- Herramientas audiovisuales.

- Herramientas de gestión de proyectos.

- Herramientas de gestión administrativa.

- Herramientas de control de gestión.

- $\quad .$. 


\section{El emprendimiento digital}

Son numerosos los autores que abordan este tema y que definen el concepto de emprendimiento digital o tecnológico. Si bien no hay diferencias sustanciales en estas definiciones, el análisis propuesto por Hull, Caisy Hung, Hair, Perotti, \& DeMartino, (Taking Advantage Of Digital Opportunities: A Typology of Digital Entreprenership, 2007) es la que mejor define las características de esta tipología de emprendimiento.

Por una parte, abordan el emprendimiento digital como una subcategoría del emprendimiento en el que se ha digitalizado parte o la totalidad de lo que sería físico en una organización tradicional.

Atendiendo a este enfoque, el grado de digitalización puede derivarse de:

- el grado de marketing digital realizado por una empresa,

- las ventas digitales de una empresa,

- la naturaleza digital del bien o servicio de una empresa,

- el potencial de distribución digital de un bien o servicio,

- las posibles interacciones digitales con agentes externos clave relacionados con la cadena de valor de la empresa,

- el potencial digital de las actividades internas asociadas con la operación de una empresa.

Esto refuerza la idea que las empresas, pueden integrar las tecnologías en cualquier función del negocio y además de manera gradual, sin necesidad de abarcar la totalidad del mismo ya que estará en función de los recursos disponibles, necesidades y tipología de cada uno de ellos.

Por otra parte, estos mismos autores proponen tres tipologías de emprendimiento digital.

En primer lugar, definen un emprendimiento digital leve, considerándolo como aquel emprendimiento que supone integrar la tecnología pero solo como un complemento de actividades empresariales más tradicionales.

La segunda tipología es la que denominan el emprendimiento digital moderado, en el que los negocios tienen un peso significativo en productos digitales, distribución digital u otros componentes digitales de la propia actividad empresarial.

Por último, está el emprendimiento digital extremo, que implica que toda la empresa es digital, incluida la producción, los bienes o servicios, la publicidad, la distribución y los clientes.

Estas tipologías de emprendimiento digital, graduadas en función de la intensidad de la tecnología en el proyecto, muestran que es posible su implantación en cualquier tipo de proyecto y que lo que seguro va a aportar es un beneficio real a aquellos negocios tradicionales que necesitan de la tecnología para ser competitivos, así como, por supuesto, en aquellos proyectos de base tecnológica donde está integrada en el propio ADN de la organización. 


\section{Conclusiones}

- Nos encontramos en un entorno socio-económico en el que las tecnologías juegan un papel fundamental en las empresas y por tanto deben estar presentes en los proyectos emprendedores desde las primeras fases su definición.

- La crisis sanitaria de la COVID-19 ha evidenciado las carencias y las necesidades de las empresas, forzando a digitalizarse de manera precipitada y en el peor de los casos, a tener que cesar el negocio por no poder seguir desarrollando su actividad "tradicional".

- Las TIC, en la actualidad son un elemento imprescindible para cualquier persona que quiera emprender un negocio, pero se debe realizar un análisis de necesidades y planificar bien su implantación. No se debe incorporar tecnología porque sí.

- "Tecnología" y "emprendimiento" están estrechamente relacionadas con la innovación. Una persona emprendedora debe desarrollar capacidades de innovación que le permitan, con el apoyo de las tecnologías, hacer si cabe su proyecto más innovador y competitivo.

- La tecnología no sólo sirve de apoyo en las diferentes funciones del negocio, sino que también sirve de complemento a los propios procesos de innovación de las empresas.

- La tecnología no es sólo materia de aquellos proyectos emprendedores de base tecnológica sino que es necesario integrarla en todos y cada uno de ellos, sobre todo en los que podemos considerar negocios "tradicionales" 


\section{Bibliografía}

- Asociación Observatorio del Emprendimiento de España (2020). Informe de Situación del emprendimiento en España ante la crisis del COVID-19

- Peirano, D., Suárez, F. (2006). TICS y empresas: propuestas conceptuales para la generación de indicadores para la sociedad de la información. Journal of information Systems and Technology Management

- Alto Comisionado para España Nación Emprendedora (2021). Informe Estrategia España Nación Emprendedora.

- Nonaka I, Takeuchi H. The Knowledge Creating Company: How Japanese Companies Create the Dynamics os Innovation. New York: Oxford University press, 1995

- Hull, C. E., Caisy Hung, Y. T., Hair, N., Perotti, V., \& DeMartino, R. (2007). Taking Advantage of Digital Opportunities. International journal of networking and virtual organisations 